Editorial

\title{
Preface to the EWOMS special issue of Human Movement Science
}

From May 31st until June 2nd, 2007, Peter Beek and his group at the Faculty of Human Movement Sciences of VU University Amsterdam hosted the third European Workshop on Movement Science (EWOMS). The aims of EWOMS are to bring together movement researchers from different disciplines, and to attract them by using a program involving appealing cutting-edge keynotes, posters and specialized mini-symposia. As reflected in its name, EWOMS has a European flavor in that the workshops are being held in Europe. Already at the start of EWOMS in 2003 in Münster, Germany (see Verwey \& Schöllhorn, 2004), the series was successful in attracting scientists from all over the world who are dedicated to the study of human and animal movement. Next, we had a very successful second workshop at the Veterinary University in Vienna, Austria in 2005 (Verwey, Schöllhorn, \& Peham, 2007). The present issue of Human Movement Science is dedicated to the third EWOMS in Amsterdam, held in 2007.

When we proposed in 2003 to Peter Beek to publish selected, high quality contributions of EWOMS in the multidisciplinary journal Human Movement Science, he, as editor-in-chief, immediately responded enthusiastically and has been very cooperative ever since. Indeed, entirely in the later spirit of EWOMS, John Whiting founded Human Movement Science in 1982 "to create a coherent and identifiable field of scientific endeavor where scientists from different disciplines meet and join arms to understand human movement in all its manifestations" (Beek, 2007). When Human Movement Science was about to celebrate its 25th anniversary, Peter proposed to dedicate EWOMS 2007 to this anniversary and he volunteered to organize the workshop in Amsterdam. EWOMS 2007 actually resulted in two special issues. The first was published in 2007 and included the 6 key note lectures presented at EWOMS in Amsterdam. These lectures were given by scientists considered forerunners in their respective fields of inquiry.

The present special issue includes selected contributions based on presentations that were given in the various mini-symposia. They were selected in view of their quality and because collectively they reflect the breadth of the movement sciences. In this issue we start off with two theoretical articles on movement control and movement skill (Torre and Wagenmakers; Schöllhorn, Mayer-Kress, Newell, and Michelbrink), to then continue with two laboratory studies on the mechanisms underlying skilled movement control (Diedrichsen and Dowling; Verwey, Abrahamse and Jiménez). Subsequently, four studies are presented of real world skills and motor activities. These include tennis (Williams, Huys, Cañal-Bruland, \& Hagemann), walking and running (Lamoth, Daffertshofer, Huys, and Beek), and horse riding (Janura, Peham, Dvorakova, and Elfmark; Witte, Schobesberger, and Peham).

The article by Torre and Wagenmakers sets out to define a research agenda by which the study of $1 / f^{\beta}$ noise can make scientific progress. From a basic discussion of the nomothetic and the mechanistic perspective, a mechanistic model of $1 / f$ noise is derived. One advantage of the suggested model resides in its successful integration with currently established models for rhythmic self-paced, synchronized, and bimanual tapping.

Schöllhorn et al. discuss major assumptions of influential approaches to the structure of variability in practice conditions from the perspective of a generalized evolving attractor landscape model of motor 
learning. A generalized model for motor learning is presented combining simulated annealing and stochastic resonance phenomena against the background of different time scales for adaptation and motor learning processes. Practical consequences of the alternative model assumptions for the structure of practice conditions are drawn, in conjunction with implications for teaching and coaching.

Diedrichsen and Dowling wonder why people are so flexible in controlling both hands, sometimes performing highly symmetric and sometimes highly asymmetric movements. To examine this, the authors use optimal control theory to predict bimanual coordination in a situation that one or two hands control a cursor and one of the hands is perturbed in a random direction. They present evidence that coordination is task-dependent, that optimal control theory can be used to model bimanual control, and that coordination arises from an optimal solution for achieving a specific distal goal.

In an attempt to understand how skill in sequential behavior is represented, Verwey et al. examined with a discrete sequence production (DSP) task, in which participants practice two fixed, short series of key presses, whether highly practiced movement skills might perhaps include a common rhythm. Based on the finding that a segmentation pattern imposed in one sequence did not transfer to a simultaneously practiced sequence or to unfamiliar sequences executed later on, they infer that segmentation is associated with a specific sequence rather than that some rhythm develops that is used with other sequences too. They further argue that effector-specific sequence learning may well be explained by a spatial representation with a hand-based reference frame.

The mechanisms underpinning anticipation of ground strokes in tennis by perturbing the dynamical information presented at different body regions are the main concern of the study by Williams et al. The results provide evidence for a model in which the skilled players reply on a more 'global' than 'local' perceptual strategy.

Even though walking and running are highly automated behaviors, it remains unclear how they are coordinated, and how transitions between those basic modes of locomotion are controlled. Lamoth et al. examined whole body gait kinematics over a wide range of treadmill speeds and compared and determined coordination patterns of walking and running using principal component analysis. From this, they conclude that walking and running entail similar, albeit speed- and gait-dependent, coordination structures, and that gait transitions bear signatures of nonequilibrium phase transitions.

The studies by Janura et al. and Witte et al. both focus on the dynamical interaction between riders and horses. Witte et al. apply principal component analysis to multidimensional kinematic data sets derived from the rider-horse system in order to identify major constituents of the three natural gaits of horses under the influence of different saddle types. The aim of the study of Janura et al. was to assess changes in the pressure distribution on a saddle during repeated hippotherapy lessons. Therefore the magnitude and distribution before and after a 5 day hippotherapeutic intervention were compared. With the growing experience of the participants, an increase was found in the pressure between the rider's body and the horses back as well as in the stability of the center of pressure movement.

At the end of this editorial, we would like to thank the members of the local organizing staff of EWOMS 2007 - especially Peter Beek, Theo de Haan, Rolf van de Langenberg, and Marjan Schot - and the other members of the scientific committee - Axel Cleeremans, Sten Grillner, Marc Jeannerod, and Gregor Schöner. We also thank the authors of the articles in this special issue. Without their effort this special issue would not have been possible. For those who may have become interested in EWOMS, the next workshop will take place in May/June 2009 in Lisbon, Portugal. We hope we have raised your interest and look forward to welcoming you there.

\section{Acknowledgement to reviewers}

We are grateful for the following scholars for the reviewing they did in the context of the present special issue:

Ramesh Balasubramaniam, University of Ottawa, Ottawa, ON, Canada

Chris Button, University of Otago, Dunedin, New Zealand

Yann Coello, Université Lille 3, Villeneuve d'Ascq, France

G. Robert Colborne, University of Bristol, Bristol, UK

Patricia de Cocq, Universtiy of Wageningen, Wageningen, The Netherlands

Till D. Frank, University of Connecticut, Storrs, CT, USA 
R. Friedrich, Westfälische Wilhelms Universität Münster, Münster, Germany

Kimberlee Jordan, University of Colorado, Boulder, CO, USA

Peter Keller, Max Planck Institute for Human Cognitive and Brain Sciences, Leipzig, Germany

Konrad Koerding, Northwestern University, Chicago, IL, USA

Julien Lagarde, Laboratory Efficience Déficience Motrices, Montpellier, France

José Luis López Rivero, Universidad Córdoba, Córdoba, Spain

Jack Murphy, University College Dublin, Dublin, Ireland

Phillipe Pourcelot, UMR INRA-ENVA, Ecole Nationale Vétérinaire d'Alfort, Maisons-Alfort, France

Michael Riley, University of Cincinnati, Cincinnati, OH, USA

Celine Robert, UMR INRA-ENVA, Ecole Nationale Vétérinaire d'Alfort, Maisons-Alfort, France

Renaud Ronsse, Katholieke Universiteit Leuven, Leuven, Belgium

Katsuyuki Sakai, University of Tokyo, Tokyo, Japan

Geert Savelsbergh, Vrije Universiteit Amsterdam, Amsterdam, The Netherlands

Thomas Schack, Bielefeld University, Bielefeld, Germany

John van der Kamp, Vrije Universiteit Amsterdam, Amsterdam, The Netherlands

René van Weeren, University of Utrecht, Utrecht, The Netherlands

Alan Wing, University of Birmingham, Birmingham, United Kingdom

\section{References}

Beek, P. J. (2007). Editorial. Human Movement Science, 26, v-vi.

Verwey, W. B., \& Schöllhorn, W. I. (Eds.) (2004). Preface to the EWOMS special issue of Human Movement Science. Human Movement Science, 23, 539-541.

Verwey, W. B., Schöllhorn, W. I., \& Peham, C. (Eds.) (2007). Preface to the EWOMS special issue of Human Movement Science. Human Movement Science, 26, 175-179.

Willem B. Verwey - Enschede, The Netherlands Wolfgang I. Schöllhorn - Mainz, Germany 